\title{
Children's and Adults' Models for Predicting Teleological Action: The Development of a Biology-Based Model
}

\author{
John E. Opfer and Susan A. Gelman
}

\begin{abstract}
Understanding that only living things must act to gain self-beneficial goals is important for developing a theorylike understanding of the living world. This research studied the models that preschoolers, fifth graders, and adults use to guide their predictions of self-beneficial, goal-directed (i.e., teleological) action. Four possible models have been suggested: finalist, complexity based, biology based, and animal based. In Study 1, participants $(N=104)$ were assigned to one of two conditions that differed in whether a beneficial or neutral object was pictured; they were asked to predict whether animals, plants, machines, and simple artifacts would modify their movement in the direction of that object. Preschoolers' predictions were consistent with an animalbased model, fifth graders' predictions were consistent with biology-based and complexity-based models, and adults' predictions were consistent with a biology-based model. Analysis of both individual response patterns and explanations supported these findings, but also showed that a significant number of preschoolers and fifth graders were finalist, and that very few individual fifth graders followed a complexity-based teleology. In Study 2, participants $(N=84)$ reported whether the animals, plants, machines, and simple artifacts in Study 1 had psychological capacities. All age groups attributed psychological capacities to animals at levels higher than other domains and at above-chance levels. The evidence from these two studies suggests that preschoolers, unlike fifth graders and adults, predict teleological action for plants and animals on the basis of these entities' inferred psychological capacities.
\end{abstract}

\section{INTRODUCTION}

According to Piaget (1969), preschoolers' causal reasoning is characterized by two related beliefs: animismthe belief that everything is alive, and finalism - the belief that everything acts goal directedly. Others have argued that preschoolers are neither animists nor finalists but that they believe only animals, plants, and microorganisms share biological properties and can act toward self-beneficial goals; that is, engage in teleological action (Keil, 1992, 1994). This article presents evidence against such a characterization of preschoolers' beliefs about teleological action, and it argues that both preschoolers' beliefs about teleological action and those about the biological domain are more restrictive than suggested by the terms finalism and animism.

Providing evidence against childhood animism, researchers have shown that preschoolers attribute properties to living things that they do not attribute to nonliving things (Carey, 1985). The research demonstrating this has studied children's attributions of growth (Hickling \& Gelman, 1995), regrowth and healing (Backsheider, Shatz, \& Gelman, 1993), nourishment (Inagaki \& Hatano, 1993), "like generating like" (Springer \& Keil, 1989), and so forth, largely, because plants and animals both engage in these kinds of activities. There is, however, a more fundamental property that all living things-not just plants and animals-share, which encompasses the activities examined in the literature. That property is teleological action (Mayr, 1982).

In the most basic sense, teleological action is any self-generated, goal-directed movement that occurs as a response to any object, state, or location that enables the actor to continue to act and exist (Binswanger, 1992; Keil, 1992). This type of goal-directed action may be observed in a variety of living things (animals, plants, micro-organisms) as well as in different levels of biological organization, from an entire organism (e.g., an animal grasping for an orange), to an organ (e.g., the heart pumping blood to itself), down to the cells (e.g., cellular respiration). Only living things are teleological in this basic sense because only living things face the alternative between death and life, which means stillness or continuing action (Binswanger, 1992).

As with action, properties may be teleological in the sense that they enable an entity to continue to exist and act (Keil, 1994; Kelemen, 1999). For example, leaves enable a plant to produce the energy it needs to survive. It is generally only through their activity, however, that these properties confer any benefit to the organism; of what value are leaves that do not react to light, wings that do not fly, or eyes that do not

(C) 2001 by the Society for Research in Child Development, Inc. All rights reserved. 0009-3920/2001/7205-0006 
see? By the same relation, understanding traits teleologically presumably requires an understanding of teleological action.

The understanding of teleological action that infants and preschoolers possess, however, has largely been assessed only by investigating their understanding of psychologically caused goal-directed action: for example, how humans modify their movement toward dubiously self-beneficial goals (Phillips \& Wellman, 1999; Spelke, Phillips, \& Woodward, 1995; Woodward, 1997) or how desires and intentions play a role in directing action (Poulin-Dubois \& Shultz, 1990; Wellman, 1990). In these studies, goal-directed action is understood not as a component in a theory of biology, but rather as a component of a theory of mind or psychology (for an alternative conception, see Gergely, Nadasdy, Csibra, \& Biro, 1995).

Neither a mind nor any psychological capacity, however, is necessary for teleological action; without a single neuron, plants survive by growing toward the sun and amoebae survive by engulfing paramecia. When such facts are understood, it is possible to have a teleological conception of all living things that is not psychological, but is domain specific, and serves as a framework to explain biological phenomena. Thus, it is reasonable to think that a biology-based teleology is a critical component of a fully formed theory of biology (Keil, 1992).

A biology-based characterization of preschoolers' teleological stance has received some empirical support. For example, when preschoolers were asked about the properties of plants and emeralds, they were more likely to state that a plant, rather than an emerald, had properties that were good for it (Keil, 1994; but see Kelemen, 1999). Past studies, however, have not examined whether preschoolers understand plants themselves to be capable of teleological action, or whether preschoolers believe that plants possess any psychological capacities that could direct their activity.

In the present study, two questions implicit in prior research were asked: what things do preschoolers believe will act teleologically, and why do they think so? The present studies differed from past research by focusing on actions and psychological capacities rather than physical properties, examining a range of domains, and asking for explanations for predictions of teleological action.

By comparing the domains for which children predict teleological action and examining their explanations, it is possible to determine the models they use to predict teleological action. For example, if children possess a biology-based model of teleological action, they should predict that living things (plants and animals) move toward self-beneficial objects but that nonliving things (machines and simple artifacts) do not. Moreover, their explanations should refer to the benefit that a goal offers to the life of the actor, rather than to mechanical forces or to the satisfaction of the actor's desires. To determine when children possess a biology-based model of teleological action, preschoolers, whose understanding of biology is disputed, and fifth graders and adults, whose understanding of biology is generally considered to involve distinct, coherent, and causal principles (Carey, 1995; Keil, 1994; Wellman \& Gelman, 1992), were tested for their beliefs about teleological action.

\section{Possible Models for Predicting Teleological Action}

The question of what domains children believe will act teleologically has been addressed by a variety of competing conceptions (Table 1). One reason for these disagreements may lie not in the dispute over children's putative theory of biology, but rather in that the question often has been posed as "What do children think is teleological?" — which leaves "is teleological" open to interpretation. For example, a pen is teleological to one who can write or make sense of what is written (see Keil, 1994; Kelemen, 1999), but the pen itself has no goal. By assessing beliefs about teleological action itself, rather than properties required for teleological action, however, researchers can address which entities are (and are not) thought to possess goals; that is, have ends toward which their action is directed. Indeed, even the meaning of "goal-directed" is often conflated with the cognate "teleological." For purposes of the present research, the term "goal-directed" was used to refer to any self-directed, contingent change in movement toward another object, state, or location (i.e., its goal); whereas "teleological" was reserved for those goal-directed movements that are en-

Table 1 Four Hypothesized Models for Predicting Teleological Action

\begin{tabular}{|c|c|c|c|c|}
\hline \multirow[b]{2}{*}{ Model } & \multicolumn{4}{|c|}{$\begin{array}{l}\text { Domains Capable of } \\
\text { Teleological Action }\end{array}$} \\
\hline & Animals & Plants & Machines & $\begin{array}{l}\text { Simple } \\
\text { Artifacts }\end{array}$ \\
\hline Finalism & $x$ & $X$ & $X$ & $x$ \\
\hline $\begin{array}{l}\text { Complexity-based } \\
\text { teleology }\end{array}$ & $X$ & $X$ & $X$ & \\
\hline $\begin{array}{l}\text { Biology-based } \\
\text { teleology } \\
\text { Animal-based } \\
\text { teleology }\end{array}$ & $X$ & $X$ & & \\
\hline
\end{tabular}


acted for the sake of the self-benefit the goal offers to the agent. This distinction enabled us to determine whether agents were thought to be capable of teleological action or goal-directed movement.

A possibility suggested by Piaget (1969) is that children expect that anything can act goal directedly, a belief known as finalism (see also Kelemen's [1999] concept of promiscuous teleology). Alternatively, Rosenblueth, Wiener, and Bigelow (1943) argued that only complex things, such as animals, plants, and machines, act toward goals, and it is plausible that children could share their view. Keil (1992) has implied that children know that all and only living things, such as plants and animals, act teleologically, whereas Carey $(1985,1995)$ implied that children believe that only animals act goal directedly (perhaps because preschoolers think that only animals have psychological capacities such as the ability to sense or desire a goal).

\section{The Present Study}

To determine which of these models (finalist, complexity based, biology based, or animal based) people hold and how they change over time, teleological prediction tasks were presented to preschoolers, fifth graders, and undergraduates (Study 1). In these tasks, participants were asked to predict whether various animals, plants, machines, and simple artifacts would act to gain a goal. In the benefit-present (BP) condition, participants were presented with scenarios in which the represented entity needed a given goal to function. In the benefit-absent (BA; control) condition, the represented entity did not need a given item to function. For example, in the benefit-present condition, participants were asked whether a plant would grow toward sunlight, whereas in the benefit-absent condition, participants were asked whether it would grow toward a picture on the wall. Thus, if participants are guided by a biology-based model of teleological action, they should predict that living things would pursue a goal in the benefit-present condition but not in the benefit-absent condition. Table 1 presents the predictions each model makes for the benefit-present condition (the concurrent prediction of no goaldirected action in the benefit-absent condition is not shown).

Additionally, participants were asked to explain why an entity would act as predicted. In these explanations, we were interested in whether they would mention biology-specific factors (as guided by a biologybased model), such as how an action benefited the life or future functioning of the entity; psychologyspecific factors (as guided by an animal-based model), such as how an action resulted from some mental state; or mechanical factors, such as how an action resulted from the entity's physical design.

In a second task (Study 2), participants were asked whether the entities in the scenarios from Study 1 possessed various psychological capacities related to goal attainment: seeing a goal, thinking, feeling pain, wanting a goal, or feeling a goal (e.g., can the plant feel sunlight?). Study 2 was designed to determine whether participants' patterns of predicting teleological action correspond with their patterns of attributing psychological capacities. That is, if participants believe that psychological capacities are necessary for teleological action, then they should claim, for example, in Study 1 that only animals act teleologically and in Study 2 that only animals possess psychological capacities; or, in Study 1, that only animals and plants act teleologically and in Study 2 that only animals and plants possess psychological capacities.

\section{STUDY 1: PREDICTIONS AND EXPLANATIONS OF TELEOLOGICAL ACTION}

\section{Method}

Participants. Thirty-two preschoolers (age: $M=5$ years, 2 months, range $=4,5-5,10 ; 17$ males, 15 females), 24 fifth graders (age: $M=10,8$, range $=10,4-$ 11,5; 11 males, 13 females), and 48 undergraduates (age: $M=19,2$, range $=18,3-23,8 ; 13$ males, 35 females) participated in this experiment. Preschoolers were enrolled in a university preschool, and fifth graders were recruited from a midwestern, suburban, public elementary school. Both schools served predominantly middle-class populations. Undergraduates were enlisted from a participant pool of volunteers enrolled in a university introductory psychology class.

Materials. Materials included $5.71 \mathrm{~cm} \times 5.71 \mathrm{~cm}$ realistic, colored line drawings of three plants, three animals, three machines, and three simple artifacts. Drawings depicted ambiguous, confabulated plants, animals, and machines. Simple artifacts, however, were drawn to represent a crayon, a basketball, and a pair of scissors to provide a baseline level of responses.

Design and procedure. Each participant was presented with 12 forced-choice prediction scenarios (Table 2). Participants were randomly assigned to one of two conditions: BP or BA. In the BP condition, the entity needed a goal to live, grow, or to continue acting, and the goal was present in the context. For example, participants were shown a picture of a plant (Figure 1) and were told, "Here is a plant growing. It needs to be near sunlight to live and grow bigger. The sunlight is over here" [window indicated]. "When this plant grows, will it grow over to where the sun- 
Table 2 Scenarios Presented in Benefit-Present (BP) and Benefit-Absent (BA) Conditions

\begin{tabular}{llll}
\hline Entity & \multicolumn{1}{c}{ Need } & \multicolumn{1}{c}{ BP Object/Goal } & BA Object/Goal \\
\hline Animal \#1 & To catch mice & A mouse & A box \\
Animal \#2 & Honey & Honey & Leaves \\
Animal \#3 & Rabbit meat inside of it & A rabbit with lots of meat in it & A ball \\
Plant \#1 & To be near sunlight & Sunlight & A picture \\
Plant \#2 & Water & Water & Oil \\
Plant \#3 & Fly juice inside of it & A fly with lots of juice in it & A pebble \\
Machine \#1 & To get more electricity & Electricity & A picture \\
Machine \#2 & Oil & Oil & Honey \\
Machine \#3 & Oil inside of it & A fish with lots of oil in it & A bag with lots of air in it \\
Simple artifact \#1 & To stay cool & Cool place & Unstained place \\
Simple artifact $\# 2$ & Air & Air & No air \\
Simple artifact $\# 3$ & To stay sharp & A sharpener & A sandwich \\
\hline
\end{tabular}

light is, or will it keep growing straight up?" As a control, in the BA condition, the needed goal was not present in the context. For example, participants were shown a picture of a plant (Figure 2) and were told, "Here is a plant growing. It needs to be near sunlight to live and grow bigger. A picture is over here" [picture indicated]. "When this plant grows, will it grow over to where the picture is, or will it keep growing straight up?" After each prediction, participants were asked to justify their response.

The order of the choices (teleological option presented first or second) was counterbalanced within subjects. The order of the domains (plants, animals, machines, simple artifacts) and the order of the entities within each domain were counterbalanced across subjects. The presentation sequence in the BP condition was identical to the sequence in the BA condition, and the sequences were identical across all age groups (preschoolers, fifth graders, and undergraduates).

\section{Results}

Each participant received four scores (0-3), indicating the number of predictions of goal-directed action made for the three entities in each domain. Thus, if participants guessed randomly, an average of 1.5 predictions of goal-directed action per domain would be expected (see Figure 3 for results).

A 3 (age) $\times 2$ (condition) $\times 4$ (domain) repeatedmeasures ANOVA was conducted on the summed scores. Post hoc analyses were conducted using the Newman-Keuls test. There was a significant main effect for age, $F(2,98)=4.34, p<.05$, in which preschoolers $(M=1.70, S D=.40)$ and fifth graders $(M=$ $1.58, S D=.31)$ were more likely than adults $(M=$ $1.31, S D=.41)$ to predict goal-directed action, $p s<.05$. There also was a significant main effect for condition, $F(1,98)=52.64, p<.001$, showing that participants were more likely to predict goal-directed action in the $\mathrm{BP}$ condition $(M=1.93, S D=.61)$ than in the BA condition $(M=1.12, S D=.55)$. There was an additional significant main effect for domain, $F(3,294)=62.15$, $p<.001$, in which goal-directed action was more likely to be predicted for animals $(M=2.34, S D=.90)$ than for plants $(M=1.52, S D=1.02)$ or machines $(M=1.39, S D=1.33), p s<.01$, which were both more likely to be predicted to act toward goals than were simple artifacts $(M=.88, S D=.97)$, $p$ s $<.01$.

In addition, there was a significant Condition $\times$ Domain interaction, $F(3,294)=8.64, p<.001$, indicating condition differences for animals (BP: $M=2.85, S D=$ .35; BA: $M=1.83, S D=.98), p<.001$; plants (BP: $M=$ $2.17, S D=.87$; BA: $M=.86, S D=.75), p<.001$; and machines (BP: $M=1.74, S D=1.24$; $\mathrm{BA}: M=1.05, S D=$ 1.07), $p<.001$, but not for simple artifacts (BP: $M=1.00$, $S D=1.09$; BA: $M=.75, S D=.83)$, ns. Furthermore, age interacted with condition, $F(2,90)=3.72, p<.05$, indicating that condition differences were greater for fifth graders (BP: $M=2.19, S D=.63$; $\mathrm{BA}: M=.98, S D=$ $.45), p<.001$, and adults (BP: $M=1.65, S D=.52$; BA: $M=.97, S D=.50) p<.001$, than for preschoolers (BP: $M=1.98, S D=.63$; BA: $M=1.42, S D=.60), p<.01$. Age also interacted with domain, $F(6,270)=8.83, p<$ .001. Finally, there was a three-way interaction among age, condition, and domain, $F(6,270)=2.22, p<.05$.

To examine this three-way interaction more closely, a 2 (condition) $\times 4$ (domain) repeated-measures ANOVA was performed on the summed scores. Post hoc analyses were conducted using the NewmanKeuls test. The results for each age group are discussed separately.

Preschoolers. There was a main effect for condition, $F(1,30)=6.74, p<.05$, indicating that preschoolers were more likely to predict goal-directed action in the BP condition than in the BA condition. There was also a main effect for domain, $F(3,90)=12.71, p<$ 
Initial State

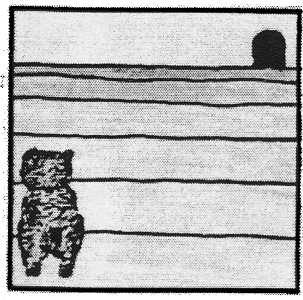

1a. Here is an animal walking straight ahead. It needs to catch mice to live and grow bigger. There is a mouse hole over there.

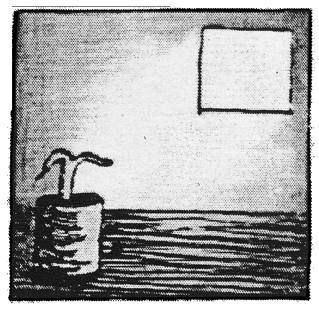

2a. Here is a plant growing. It needs to be near sunlight to live and grow bigger. The sunlight is over here.

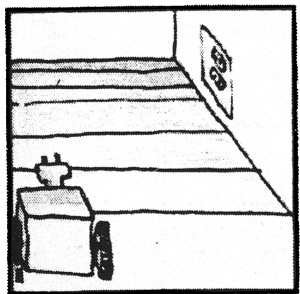

3a. Here is a machine rolling straight ahead. It needs to get more electricity to roll and work. The electricity is over here.

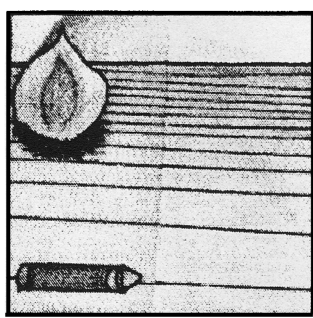

4a. Here's something rolling straight ahead. It needs to stay cool so it doesn't melt and fall apart. Over here it's cool, and over here it's very hot.
Goal-Directed Action

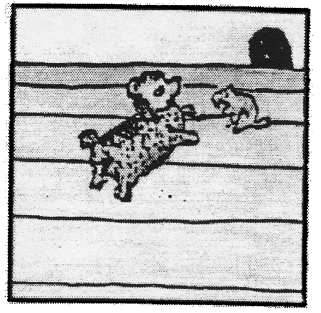

$1 \mathrm{~b}$. When the mouse comes out of its hole, will the animal move over to where the mouse is,

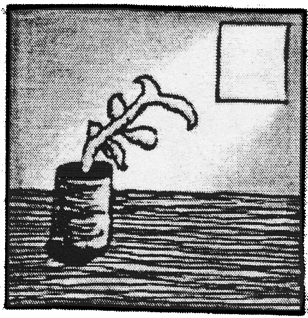

$2 \mathrm{~b}$. When this plant grows, will it grow over to where the sunlight is,

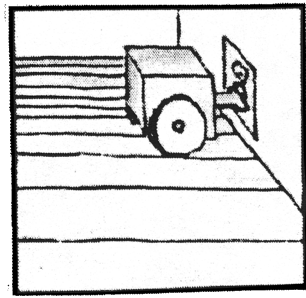

$3 \mathrm{~b}$. When this machine rolls further, will it roll over to where the electricity is,

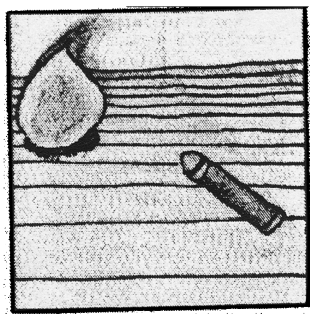

$4 \mathrm{~b}$. When this thing rolls further, will it roll over to where the cool place is,
Non-Goal-Directed Action

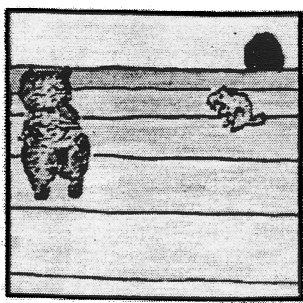

1c. or will the animal keep moving straight ahead?

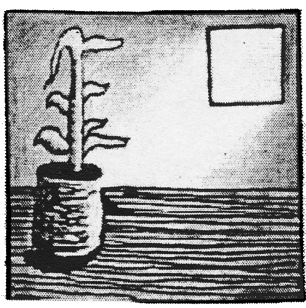

2c. or will it keep growing straight up?

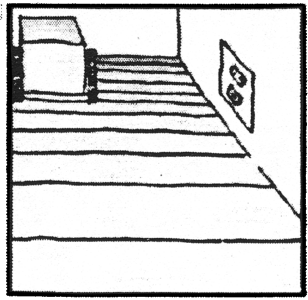

3c. or will it keep rolling straight ahead?

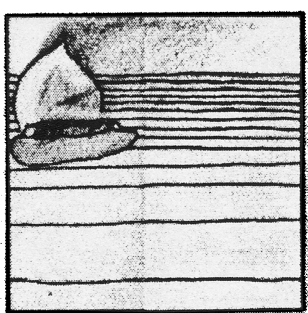

4c. or will it keep rolling straight ahead?

Figure 1 Illustration of selected items from benefit-present condition. 
Initial State

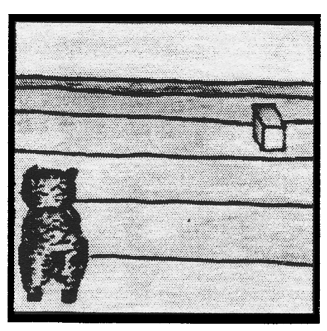

1a. Here is an animal walking straight ahead. It needs to catch mice to live and grow bigger. There is a box over here.

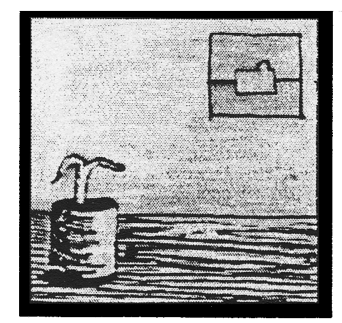

2a. Here is a plant growing. It needs to be near sunlight to live and grow bigger. A picture is over here.

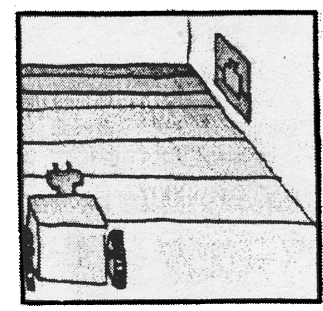

3a. Here is a machine rolling straight ahead. It needs to get more electricity to roll and work. A picture is over here.

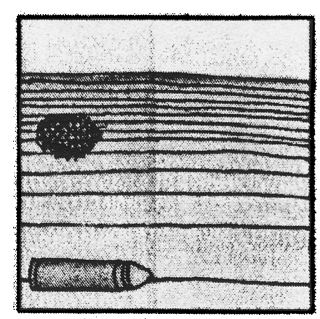

4a. Here's something rolling straight ahead. It needs to stay cool so it doesn't melt and fall apart. Over here there's a stain; over here there's not.
Goal-Directed Action

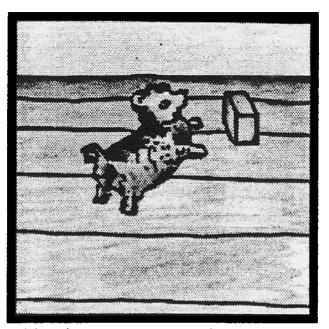

1b. Will the animal move over to where the box is,

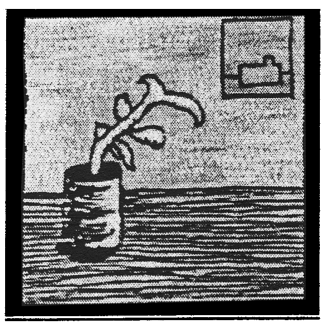

$2 b$. When this plant grows, will it grow over to where the picture is,

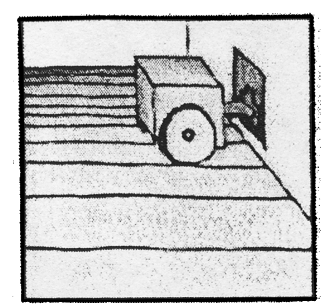

$3 b$. When this machine rolls further, will it roll over to where the picture is,

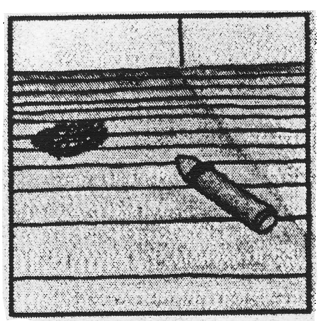

4b. When this thing rolls further, will it roll over to here,
Non-Goal-Directed Action

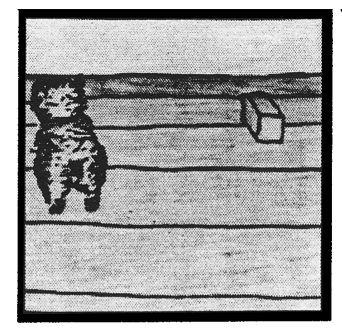

1c. or will the animal keep moving straight ahead?

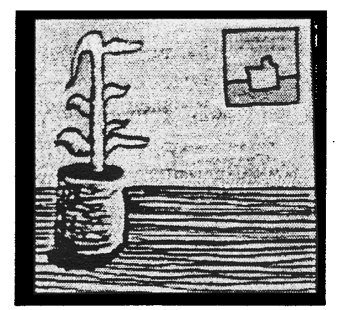

2c. or will it keep growing straight up?

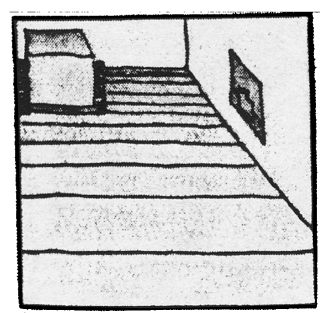

3c. or will it keep rolling straight ahead?

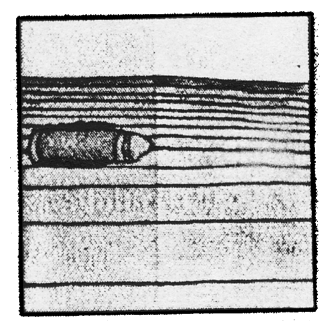

4c. or will it keep rolling straight ahead?

Figure 2 Illustration of selected items from benefit-absent condition. 


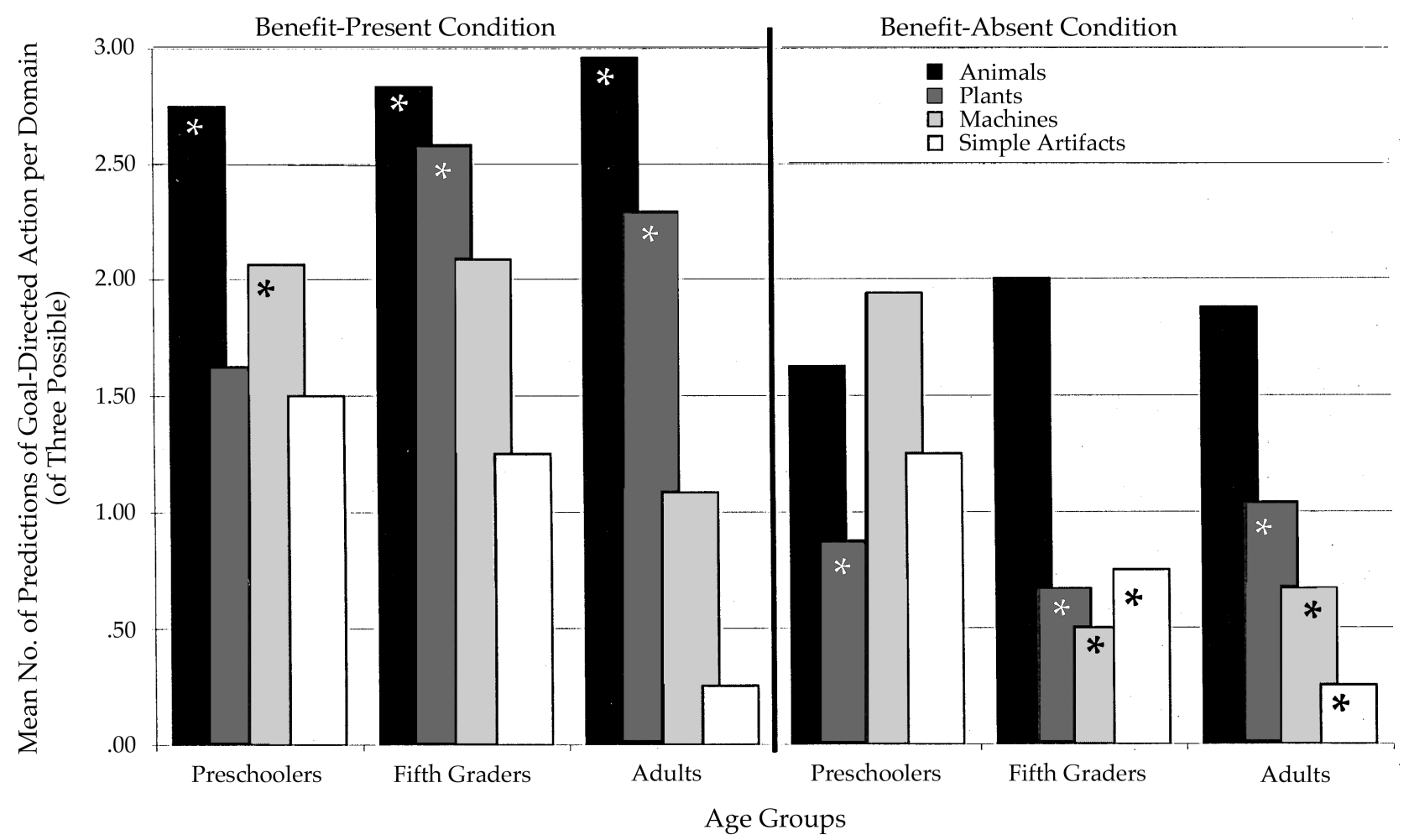

Figure 3 Predictions of goal-directed action by age, domain, and condition. ${ }^{*} p<.05$ (versus chance).

.001 , indicating that preschoolers were more likely to predict goal-directed action for animals $(M=2.19$, $S D=.51)$ and machines $(M=2.00, S D=.63)$ than for plants $(M=1.25, S D=.51)$ or simple artifacts $(M=$ $1.38, S D=.72), p s<.01$. There was also a Condition $\times$ Domain interaction, $p<.05$. That is, preschoolers were more likely to predict that animals would act toward beneficial goals than neutral ones (BP: $M=2.75$, $S D=.45 ; \mathrm{BA}: M=1.75, S D=.89), p<.001$, as they were for plants (BP: $M=1.62, S D=.96$; BA: $M=.88$, $S D=.72), p<.05$, but not machines (BP: $M=2.06, S D=$ .93; BA: $M=1.94, S D=1.06), n s$, or simple artifacts (BP: $M=1.50, S D=.97$; BA: $M=1.25, S D=.93$ ), $n s$. Within the BP condition, preschoolers predicted that animals would act teleologically at levels greater than expected by chance, $p<.001$, and they were more likely to predict that animals would act toward beneficial goals (i.e., teleologically) than would any other domain, $p$ s $<.01$; preschoolers' predictions for plants, machines, and simple artifacts did not differ. Within the BA condition, preschoolers predicted goal-directed action for animals, machines, and simple artifacts at chance levels, and they predicted goal-directed action for plants at below-chance levels, $p<.01$.

Fifth graders. There was a main effect for condition, $F(1,22)=29.32, p<.001$, indicating that fifth graders were significantly more likely to predict goal-directed action in the BP condition than in the BA condition. There was also a significant main effect for domain, $F(3,66)=14.31, p<.001$, indicating that fifth graders were more likely to predict goal-directed action for animals $(M=2.42, S D=.42)$ than for plants $(M=$ $1.63, S D=.57)$, machines $(M=1.29, S D=.45)$, or simple artifacts $(M=1.00, S D=.64), p \mathrm{~s}<.01$, and that they were more likely to predict teleological action for plants and machines than for simple artifacts, $p s<.05$. Finally, condition interacted with domain, $F(3$, $66)=4.10, p<.01$, indicating condition differences in predictions of goal-directed action for animals (BP: $M=2.83, S D=.39$; BA: $M=2.00, S D=.85), p<.05$, plants (BP: $M=2.58, S D=.67$; BA: $M=2.00, S D=$ .77 ), $p<.001$, and machines (BP: $M=2.08, S D=1.16$; $\mathrm{BA}: M=.50, S D=.80), p<.001$, but not for simple artifacts (BP: $M=1.25, S D=1.29$; BA: $M=.75, S D=$ $.75), n s$. In the BP condition, fifth graders were equally likely to predict that animals, plants, and machines would act teleologically, more so than would simple artifacts, $p \mathrm{~s}<.05$. Further, in the BP condition, fifth graders were more likely than expected by chance to predict that animals and plants would act teleologically, $p s<$ .001 , whereas their predictions for machines were at chance levels. In the BA condition, fifth graders were 
more likely to predict goal-directed action for animals than for plants, machines, or simple artifacts, $p \mathrm{~s}<.01$.

Adults. There was a main effect for condition, $F(1,46)=21.26, p<.001$, indicating that adults were significantly more likely to predict goal-directed action in the BP condition than in the BA condition. There was also a main effect for domain, $F(3,138)=$ $75.24, p<.001$, indicating that adults were more likely to predict goal-directed action for animals $(M=2.42$, $S D=.55)$ than for plants $(M=1.67, S D=.52), p<.01$, for plants than for machines $(M=.90, S D=.81), p<.05$, and for machines than for simple artifacts $(M=.25$, $S D=.42), p<.01$. Finally, condition interacted with domain, $F(3,138)=4.17, p<.001$, indicating condition differences in predictions of goal-directed action for animals (BP: $M=2.96, S D=.20$; BA: $M=1.88, S D=1.12$ ), $p<.001$, and plants (BP: $M=2.29, S D=.75$; BA: $M=$ $1.04, S D=.75), p<.001$, but not for machines (BP: $M=$ $1.08, S D=1.28$; BA: $M=.71, S D=.81)$, $n s$, or simple artifacts (BP: $M=.25, S D=.68$; BA: $M=.25, S D=$ $.53), n s$. In the BP condition, adults were more likely than expected by chance to predict that living things would act teleologically, $p<.001$ for animals, $p<.05$ for plants, but were below chance in their predictions that simple artifacts would do so, $p<.001$. In the BA condition, adults were more likely to predict goal-directed action for animals than for plants, machines, or simple artifacts, $p$ s $<.01$.

Summary. In comparing the hypothesized models for predicting teleological action (Table 1) with the age groups' performance, it appears that preschoolers predominantly followed an animal-based model for predicting teleological action (i.e., changes in action for a self-beneficial goal). In contrast, in the BA (control) condition, preschoolers predicted the analogous action at or below chance levels. Fifth graders' performance, on the other hand, suggests that they used both complexity-based and biology-based models in predicting teleological action, whereas in the BA condition, they were unlikely to predict that nonanimals would engage in the analogous action. Adults seemed to follow a biology-based model for predicting teleological action and were also unlikely to predict that nonanimals would engage in goal-directed action.

Individual response patterns. Because averaging age group performances can mask important developmental differences (Siegler, 1996), participants' predictions in the two conditions were also analyzed by looking at individual response patterns. Participants' predictions received a high score when two or three of the three possible entities in each of the four domains were predicted to act toward a goal; and a low score was assigned when zero or one of the three possible entities were predicted to act toward a goal. Thus, there were a total of 16 possible response patterns $\left(2^{4}\right)$. Five of these were examined, which were glossed as finalism (high in all domains), complexity based (high for animals, plants, and machines only), biology based (high for animals and plants only), animal based (high for animals only), and no predictions (not high for any domain). The frequency of each response pattern was compared with chance by performing a sign test. The results are presented in Table 3. Nearly all the participants fell into one of these five response patterns. In the BP condition, preschoolers' predictions were consistent with animal-based and finalist models. In contrast, fifth graders' predictions were consistent with biology-based and finalist models, and adults' predictions were consistent with biology-based and complexity-based models. These results, however, should be viewed with caution, given the small number of participants in each cell.

Explanations. Explanations of goal-directed action were also coded and tabulated in both conditions.

Table 3 Individual Response Patterns in Predicting Teleological Action

\begin{tabular}{|c|c|c|c|c|c|c|}
\hline \multirow[b]{3}{*}{ Response Pattern } & \multicolumn{6}{|c|}{ Proportion of Participants Following Each Response Pattern } \\
\hline & \multicolumn{3}{|c|}{ Benefit-Present Condition } & \multicolumn{3}{|c|}{ Benefit-Absent Condition } \\
\hline & $\begin{array}{l}\text { Preschoolers } \\
\quad(n=16)\end{array}$ & $\begin{array}{l}\text { Fifth Graders } \\
\quad(n=12)\end{array}$ & $\begin{array}{l}\text { Adults } \\
(n=24)\end{array}$ & $\begin{array}{l}\text { Preschoolers } \\
\quad(n=16)\end{array}$ & $\begin{array}{l}\text { Fifth Graders } \\
\quad(n=12)\end{array}$ & $\begin{array}{c}\text { Adults } \\
(n=24)\end{array}$ \\
\hline Finalism & $.31^{\star *}$ & $.42^{* * *}$ & .04 & .06 & .00 & .00 \\
\hline Biology based & .06 & $.33^{* *}$ & $.50^{* * *}$ & .06 & .06 & .08 \\
\hline Complexity based & .13 & .13 & $.29^{* * *}$ & .06 & .00 & .04 \\
\hline Animal based & $.25^{\star *}$ & .06 & .17 & .06 & $.42^{* * *}$ & $.38^{\star * *}$ \\
\hline No predictions & .00 & .00 & .00 & .19 & .17 & $.33^{* * *}$ \\
\hline Other & .25 & .00 & .00 & .56 & .33 & .13 \\
\hline
\end{tabular}

Note: Totals may not sum to 1.00 due to rounding.

${ }^{* *} p<.01 ;{ }^{* * *} p<.001$. 
Participants typically identified the relation between the goal and the entity, but explanations differed in what participants perceived to be the benefit of the goal. For example, some participants mentioned whether the entity needed the goal to live, to survive, or to keep working (ultimate explanations). Additionally, participants differed in what they perceived was directing the movement of the entity. For example, some participants referred to a psychological state directing the movement of the entity, as in "Because he wanted it" (psychological explanations). Others referred to some design or mechanical force directing the movement of the entity, as in, "It looked like [the crayon] was already rolling in that direction" (mechanical explanations). Each explanation could be categorized as more than one type. For example, one preschooler explained that an animal would move toward a mouse "'cause it doesn't like (psychological) to run away from things: it likes (psychological) to catch things so it can keep living (ultimate)." Thus, this explanation was coded as both psychological and ultimate. Of 481 explanations, 61 were randomly selected and coded by another researcher; interrater reliability was $92 \%, \mathrm{\kappa}=.88$.

An adjusted total of each explanation type was calculated for the predictions of goal-directed action. For example, if a participant predicted that 6 of 12 entities would act teleologically in the BP condition, then the number of explanations per explanation type was multiplied by two so that the total number of predictions for a given domain did not load on any given explanation type.

To compare the explanation types used by each age group, a 3 (age): $\times 3$ (explanation type): $\times 2$ (condition) ANOVA was conducted on the adjusted totals of explanations per set of predictions. All post hoc analyses were conducted using the Newman-Keuls test. There was a main effect for condition, $F(1,74)=9.93$, $p<.005$, indicating that more of the explanation types were offered in the BP than in the BA condition. Additionally, there was a three-way interaction, $F(4,148)=$ 5.11, $p<.001$. To examine this interaction more closely, each condition is discussed separately.

In the BP condtion, there was a main effect for explanation, $F(2,74)=12.77, p<.001$, indicating that ultimate $(M=3.55, S D=3.11)$ and psychological $(M=3.57$, $S D=2.89$ ) explanations were preferred to mechanical $(M=.84, S D=2.25)$ ones, but there was no main effect for age. There also was a significant interaction between age and explanation, $F(4,74)=2.72, p<.05$. Fifth graders and adults were more likely than preschoolers to provide ultimate explanations (preschoolers: $M=1.48, S D=2.19$; fifth graders: $M=4.88, S D=3.68$; adults: $M=4.28, S D=2.39$ ), $p \mathrm{~s}<.01$, but there were no significant age differences for other explanation types. Preschoolers were more likely to provide psychological $(M=3.72, S D=3.07)$ explanations than ultimate $(M=1.48, S D=2.19)$ or mechanical $(M=1.18$, $S D=1.52)$ ones, $p \mathrm{~s}<.05$, whereas fifth graders and adults were more likely to provide ultimate (fifth graders: $M=4.88, S D=3.68$; adults: $M=4.28, S D=$ 2.39) and psychological (fifth graders: $M=3.73, S D=$ 3.32; adults: $M=3.06, S D=3.22)$ explanations than mechanical (fifth graders: $M=.43, S D=.65$; adults: $M=$ $.93, S D=1.30)$ ones, $p$ s $<.01$.

In the BA condition, there was a main effect for age, $F(2,74)=4.12, p<.05$, indicating that adults $(M=2.60, S D=1.28)$ were more likely to provide ultimate, psychological, and mechanical explanations than were preschoolers $(M=1.37, S D=1.29)$ or fifth graders $(M=1.34, S D=1.28)$. There also was a main effect for explanation type, $F(2,74)=22.25, p<.001$, indicating that more psychological explanations $(M=$ $3.87, S D=4.08)$ were offered than either ultimate $(M=.32, S D=.93)$ or mechanical $(M=1.13, S D=1.54)$ ones, $p$ s $<.01$, which did not differ. Further, there was an Age $\times$ Explanation Type interaction, $F(4,74)=4.39$, $p<.005$. Age groups did not differ in how often they offered ultimate (preschoolers: $M=.64, S D=1.04$; fifth graders: $M=.33, S D=1.15$; adults: $M=.00, S D=$ .00 ) and mechanical (preschoolers: $M=.89, S D=1.47$; fifth graders: $M=1.26, S D=1.37$; adults: $M=1.24$, $S D=1.85)$ explanations, but adults were more likely to offer psychological explanations $(M=6.57, S D=$ 4.66) than were either fifth graders $(M=2.46, S D=3.52)$ or preschoolers $(M=2.59, S D=3.01), p s<.01$. Additionally, both adults and preschoolers reliably preferred psychological explanations to ultimate and mechanical ones, $p \mathrm{~s}<.01$, whereas fifth graders did not demonstrate any reliable preferences, $p=.11$.

Discussion. In predicting teleological action, preschoolers, fifth graders, and adults were sensitive to differences in the domain of the entity and whether the entity needed the goal to continue acting. As a group, preschoolers' responses were consistent with an animal-based model, fifth graders' responses were consistent with both biology-based and complexitybased models, and adults' responses were consistent with a biology-based model. These findings are largely consistent with the individual response patterns observed: (1) preschoolers typically fell into the animal-based or finalist models; (2) fifth graders were likely to follow biology-based or finalist models, and (3) adults primarily followed the biology-based model. Additionally, participants' explanations seemed consistent with the pattern of predictions observed. Just as preschoolers were likely to claim that only animals would act teleologically, so were they more 
likely to provide psychological explanations than any other explanations. Further, just as fifth graders and adults were more likely than preschoolers to predict that plants and animals would act teleologically, so were they more likely than preschoolers to provide ultimate explanations than mechanical explanations of teleological action.

\section{STUDY 2: ATTRIBUTIONS OF PSYCHOLOGICAL CAPACITIES}

Given that preschoolers were likely to follow an animal-based model, do they think that animals act teleologically because they believe that only animals possess psychological capacities? Although some studies have reported that preschoolers believe that plants and microorganisms do not possess the capacity to think (Gutheil, Vera, \& Keil, 1998; Keil, 1992), thinking is only one among a variety of psychological capacities that might be considered necessary for teleological action. Additionally, a number of studies have found that preschoolers vary widely in how likely they are to attribute psychological capacities to animals and in what psychological capacities they attribute to them. Indeed, often they are less likely than older children and adults to attribute psychological capacities to animals. For example, although preschoolers through adults seem especially likely to attribute emotional capacities to mammals, preschoolers have been less likely than older children to attribute either cognitive or emotional capacities to fish, insects, and worms (Carey, 1985; Coley, 1995; Solomon \& Cassimitis, 1999). Because some of the animals presented to the children in Study 1 were not clear-cut representations of any of these classes of animals, and because the full scope of children's psychological constructs of various plants has not been addressed in the literature, we thought it important to examine children's beliefs about the various psychological capacities of the novel animals, plants, machines, and simple artifacts presented in Study 1.

Indeed, even adults may believe that some psychological capacities are necessary for teleological action, but they may differ from preschoolers in which capacities they believe are necessary. For example, fifth graders and adults predict that plants will act teleologically, but they may believe that plants are so capable because they believe that plants possess the capacity to feel goals, but not the capacity to think about goals (as preschoolers might).

To address whether children and adults differ in the psychological capacities they attribute to entities, psychological attribution tasks were presented to preschoolers, fifth graders, and undergraduates. Partici- pants were asked about the same animals, plants, machines, and simple artifacts portrayed in Study 1. It was anticipated that all participants would attribute psychological capacities only to animals.

\section{Method}

Participants. Twelve preschoolers (age: $M=5,0$, range $=4,6-5,4 ; 8$ males, 4 females), 24 fifth graders (age: $M=10,8$, range $=10,4-11,5 ; 11$ males, 13 females), and 48 undergraduates (age: $M=19,2$, range = $18,3-23,8 ; 13$ males, 35 females) participated in this study. Preschoolers were enrolled in a university preschool serving a predominantly middle-class population. The undergraduates and fifth graders were the same participants as in Study 1; Study 2 immediately followed the administration of Study 1 . Due to the length of Studies 1 and 2, none of the preschoolers in Study 1 participated in Study 2, to avoid the length of the first study interfering with performance in the second.

Materials. The materials used in Study 2 were the same as those used in Study 1.

Design and procedure. Each participant was presented with 60 questions (five questions per entity, three entities per domain, four domains) about the psychological capacities of the entities. For each entity, the domain was identified (e.g., "This is a plant"), and participants were asked "Can it see [the goal]?" "Can it think?" "Can it feel pain?" "Can it want anything?" and "Can it feel [the goal]?"

The order of the domains was counterbalanced, and the order of the capacities (see, think, feel pain, want, and feel) within each domain was randomized. The presentation sequences were identical across all age groups.

\section{Results}

Each participant received four scores ( 3 entities $\times 5$ psychological capacities $=0-15)$, indicating the number of psychological attributions made within each domain (Figure 4).

A three (age) $\times 4$ (domain) $\times 5$ (capacity) repeatedmeasures ANOVA was conducted on the summed scores for each capacity considered separately. Because there were three opportunities to attribute a given psychological capacity for each domain, chance responding should yield 1.5 attributions. Post hoc comparisons were performed using the NeumanKeuls test. There was no significant main effect for age, $p=.15$. There was, however, a significant main effect for domain, $F(3,246)=348.24, p<.001$, indicat- 


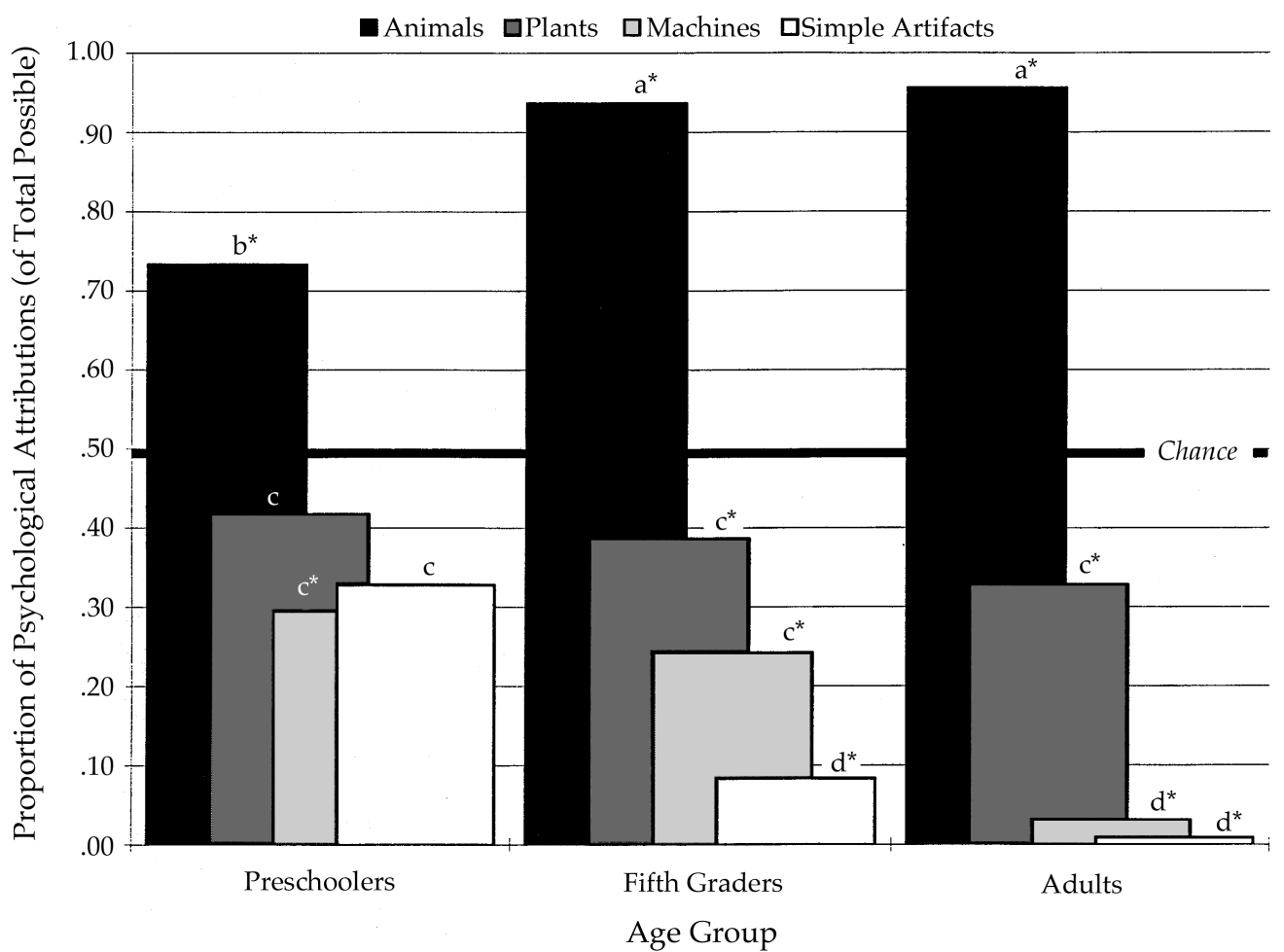

Figure 4 Attributions of psychological capacities by age and domain. ${ }^{*} p<.05$ (versus chance); Fisher comparisons: $p(a \neq b \neq c \neq d)<.05$.

ing that participants were more likely to attribute psychological capacities to animals than to plants, machines, and simple artifacts, $p \mathrm{~s}<.01$; were more likely to attribute psychological capacities to plants than to machines and simple artifacts, $p \mathrm{~s}<.01$; and were more likely to attribute psychological capacities to machines than to simple artifacts, $p<.05$. For all age groups, animals were the only domain to which psychological capacities were attributed at abovechance levels, $p \mathrm{~s}<.05$. There was a significant Age $\times$ Domain interaction, $F(6,246)=18.22, p<.001$, indicating that domain differences were greater with increasing age. There was a main effect for capacity, $F(4,328)=58.05, p<.001$, indicating that participants were more likely to attribute feeling $(M=1.74, S D=$ 1.33) than any other capacity, $p s<.01$, to attribute wanting $(M=1.43, S D=1.40)$ more than feeling pain $(M=1.02, S D=1.35)$, thinking $(M=.91, S D=1.31)$, or seeing $(M=.85, S D=1.24), p s<.01$; and to attribute feeling pain more than seeing, $p<.05$. There was no significant Age $\times$ Capacity interaction, $p=.33$. Capacity, however, interacted with domain, $F(12,984)=$ $22.45, p<.001$, indicating that capacity differences were greater for some domains than others. Finally, there was a significant Age $\times$ Domain $\times$ Capacity interaction, $F(24,984)=6.03, p<.001($ Table 4$)$.
To determine which domains were said to possess which psychological capacities, each capacity in each domain was compared with chance using one-group $t$ tests. Preschoolers attributed only seeing $(M=2.42$, $S D=.79), p<.005$, feeling $(M=2.75, S D=.45), p<$ .001 , and wanting $(M=2.17, S D=1.03), p<.05$, to animals at above-chance levels, and they did not attribute any psychological capacities to plants at above-chance levels. Adults and fifth graders attributed all capacities to animals at above-chance levels, $p \mathrm{~s}<.001$. Interestingly, adults and fifth graders also attributed feeling to plants at above-chance levels (fifth graders: $M=2.38, S D=.88$; adults: $M=2.41$, $S D=.77), p s<.001$.

\section{Discussion}

Interpreting an animal-based model as psychology based and a biology-based model as nonpsychology based depends on the assumption that animals are viewed as having psychological attributes and that plants are not. Study 2 tested this assumption. We found that when various psychological capacities (feeling, wanting, feeling pain, thinking, and seeing) were collapsed, all age groups attributed psychological capacities only to animals, which is consistent 
Table 4 Mean Attributions of Psychological Capacities per Domain (of Three Possible)

\begin{tabular}{lcccc}
\hline $\begin{array}{l}\text { Psychological Capacity/ } \\
\text { Participants }\end{array}$ & Animals & Plants & Machines & Simple Artifacts \\
\hline Seeing & & & \\
$\quad$ Preschoolers & $2.42(.79)^{*}$ & $.17(.39)^{*}$ & $.17(.39)^{*}$ & $.17(.39)^{*}$ \\
Fifth graders & $2.67(.76)^{*}$ & $.29(.62)^{*}$ & $.42(.65)^{*}$ & $.08(.41)^{*}$ \\
Adults & $2.81(.53)^{*}$ & $.27(.71)^{*}$ & $.10(.42)^{*}$ & $.04(.20)^{*}$ \\
Thinking & & & & \\
Preschoolers & $1.58(1.24)$ & $1.17(1.27)$ & $.58(1.16)^{*}$ & $.75(1.14)^{*}$ \\
Fifth graders & $2.88(.61)^{*}$ & $.42(.83)^{*}$ & $.50(.88)^{*}$ & $.04(.20)^{*}$ \\
Adults & $2.94(.43)^{*}$ & $.31(.85)^{*}$ & $.00(.00)^{*}$ & $.02(.14)^{*}$ \\
Feeling pain & & & & \\
Preschoolers & $2.08(1.00)$ & $1.33(1.15)$ & $.67(1.15)^{*}$ & $1.17(1.34)$ \\
Fifth Graders & $2.88(.61)^{*}$ & $.88(1.33)^{*}$ & $.00(.00)^{*}$ & $.08(.28)^{*}$ \\
Adults & $2.94(.43)^{*}$ & $.60(1.12)^{*}$ & $.02(.14)^{*}$ & $.02(.14)^{*}$ \\
Wanting & & & & \\
Preschoolers & $2.17(1.03)^{*}$ & $1.83(1.27)$ & $1.25(1.36)$ & $1.25(1.36)$ \\
Fifth graders & $2.79(.66)^{*}$ & $1.88(1.36)$ & $1.25(1.29)$ & $.42(.93)^{*}$ \\
Adults & $2.94(.43)^{*}$ & $1.46(1.40)$ & $.33(.88)^{*}$ & $.13(.39)^{*}$ \\
Feeling & & & \\
Preschoolers & $2.75(.45)^{*}$ & $2.08(1.00)$ & $1.75(1.29)$ & $1.58(1.31)$ \\
Fifth graders & $2.83(.64)^{*}$ & $2.38(.88)^{*}$ & $1.46(1.06)$ & $.63(1.01)^{*}$ \\
Adults & $3.00(.00)^{*}$ & $2.46(.77)^{*}$ & $.44(.87)^{*}$ & $.21(.65)^{*}$ \\
\hline
\end{tabular}

Note: Values in parentheses are standard deviations.

${ }^{*} p<.05$, two-tailed $t$ test versus chance (1.5).

with many previous reports (Carey, 1985; Gutheil, Vera, \& Keil, 1998; Solomon \& Cassimatis, 1999). When individual psychological capacities were analyzed, however, we found that animals were not the only type of entity that was attributed as having psychological capacities. That is, fifth graders and adults reliably attributed feeling to plants and animals, but not to machines or simple artifacts.

\section{GENERAL DISCUSSION}

An abstract, principled understanding of the living world is an important achievement. This understanding would enable one to treat plants and animals as equivalent in important respects, including the capacity to act teleologically. It is probably no coincidence, therefore, that developmentalists' characterizations of children's biological reasoning is often the same as their characterization of their teleological reasoning (Carey, 1985; Keil, 1992; Piaget, 1969). The present study sought to discover what kind of teleological understanding preschoolers possess by looking at their pattern of predictions of teleological action, their explanations for these predictions, and their attributions of psychological capacities, to understand how they may constrain teleological predictions.

Although preschoolers' teleological understanding has variously been characterized as finalist and biology based, the present findings suggest that the dominant models used by preschoolers are finalist and animal based (Study 1). The use of each of these warrants some explanation.

First, the finalist pattern of predictions among preschoolers and fifth graders was seen both in their group performance, in which both groups of children overall were more likely than adults to predict goaldirected action (irrespective of domain or condition), and in their individual prediction patterns, in which a significant number of both age groups stated that all entities would act teleologically (Table 3 ). These results replicate findings by Kelemen (1999) and Piaget (1969), who concluded that preschoolers generally expect that all kinds of things can act toward goals or are designed to accomplish such goals. In this view, development entails preschoolers rejecting a promiscuous teleology in favor of a selective one. Another possibility, however, is that preschoolers' finalism is not their initial model but rather an intermediate one. This would suggest that preschoolers initially expect that only (psychological) animals will act teleologically, but that when they also learn that plants can do so, they then overgeneralize this capacity to other nonpsychological entities before understanding that the life of plants and animals requires goal-directed actionthis later understanding would then logically exclude nonliving machines and simple artifacts as teleological actors. Some support for this explanation may be seen in the individual response patterns of fifth graders, who 
seemed to abandon the animal-based model in favor of biology-based and finalist models (Table 3). Whether this alternative proposal is correct requires further research on the conditions that produce finalism.

Second, it is difficult to reconcile the present findings with characterizations of preschoolers as having a biology-based model of teleological action. Contrary to this characterization, virtually no individual preschoolers followed the biology-based model (Table 3), and, as a group, they were more likely to predict teleological action for animals than for plants, machines, or simple artifacts; that is, they predicted that only animals would act teleologically at above-chance levels (Figure 3). It appears that this animal-based model is constrained by two beliefs: (1) that an entity must sense or desire a goal before it can direct its movement toward that goal (Study 1), and (2) that animals are the most likely type of entity to possess such psychological capacities (Study 2).

Why do many preschoolers consider animals unique in this respect? To answer this question, it should be noted that preschoolers' patterns of teleological predictions were mirrored in their attributions of psychological capacities (Study 2), and that their explanations of teleological actions most frequently referred to psychological states (Study 1). At times, this psychologybased model even violated their pattern of psychological attributions. As one example (of several), when asked to justify his prediction that a machine would move toward a picture on the wall, one preschooler responded, "because [the machine] will think it's electricity." Given this pattern of predictions, explanations, and attributions, it seems reasonable to conclude that preschoolers believe goal-directed action to be a consequence of psychological states induced by beneficial objects.

Of course, the link between the predictions in Study 1 and the attributions in Study 2 is correlational. Therefore, it is possible that preschoolers attributed psychological capacities on the basis of teleological action (the teleology-first hypothesis) rather than vice versa (the psychology-first hypothesis). Following the teleology-first hypothesis, preschoolers were simply ignorant that plants could act teleologically (Study 1) and so failed to attribute psychological capacities to them (Study 2). If this were the case, then it should be expected that preschoolers would attribute psychological capacities to plants and other insentient entities upon evidence that these entities act teleologically. The present research does not address this possibility, but the supposition is that preschoolers can attribute psychological capacities on the basis of nonteleological factors, and that they use these psychological judgments to constrain their pre- dictions of teleological action. If this is the case, then it would be expected that preschoolers would not be especially predisposed to attribute psychological capacities to plants and other insentient entities, even with evidence that these entities act teleologically.

Further, barring the psychology-first hypothesis, there appears to be no way to explain preschoolers' explanations. That is, preschoolers were presented with a thing that acted nonteleologically (e.g., an animal moving away from a mouse it needed to eat, or a plant growing away from the sunlight it needed to grow) and they were asked to predict whether it would change its non-goal-directed movement toward the beneficial goal. At that point, there was no teleological action on which preschoolers could base their psychological judgments, as the teleology-first hypothesis would require. Therefore, when preschoolers explained that entities would act teleologically because they possessed various psychological states (Study 2), they could not have done so on the basis of any a posteriori beliefs about teleological action. In contrast, preschoolers could have readily explained their predictions of goal directedness on the basis of their a priori beliefs about psychological capacities.

In contrast to preschoolers' psychology-based model, fifth graders and adults, who are generally credited with possessing a principled understanding of biology (Carey, 1995; Keil, 1994), demonstrated a biology-based model of teleological action. First, they were likely to predict that both plants and animals would act toward goals, and they did so more often in the BP condition (in which survival was at stake) than in the BA condition (in which survival was unaffected by the action). Supporting this developmental progression, one third of fifth graders and one half of adults in the BP condition fell into the biology-based model, whereas only one preschooler did. Further, fifth graders and adults were likely to predict teleological action for plants (Study 1), for which they generally were not likely to attribute psychological capacities (Study 2). Finally, fifth graders and adults often referred to the life or survival of the entity when explaining its actions, whereas preschoolers rarely did so (Study 1, Explanations). Given this pattern of predictions, explanations, and attributions, it seems reasonable to conclude that fifth graders and adults believe teleological action to be a critical part of being alive.

Although fifth graders and adults may believe teleological action to be a consequence of being alive, their predictions and explanations of teleological action took both biological and psychological facts into account. When asked to justify the prediction that an animal would move toward a box, for example, a fifth 
grader responded that the animal would move toward the box "to see what's in there, and if there's nothing he'll just go right on." An adult responded, "because, like, animals have a natural instinct to be curious." Interestingly, fifth graders and adults were also likely to attribute feeling to plants, but not to machines and simple artifacts. It is possible, then, that fifth graders and adults believe that the capacity to feel is involved even in the teleological actions of plants; however, it is also possible that they simply use the term "feel" as a metaphor in the biological domain and that this metaphor seems apt when explaining movement toward beneficial objects.

Although fifth graders and adults were more likely to claim that animals would pursue beneficial objects than neutral ones, they were also more likely to predict that animals would pursue neutral objects than would any other domain. It seems likely that fifth graders and adults treated the BA condition as a kind of false-belief task, a widely used index of children's theory of mind that depicts an actor whose pursuit of a goal is disrupted by the invisible displacement of that goal (Wellman, 1990). As in the false-belief task, some participants interpreted the neutral object as a mistakenly suspected cover for a beneficial goal. One fifth grader, for example, explained that an animal that needed honey would move to a log filled with leaves "'cause maybe leaves fell in the log, and there's honey inside." It remains unclear, however, why 5year-old preschoolers-who typically succeed on false-belief tasks (Wellman, 1990)—were as likely to think that animals would be "fooled" as they would machines.

It is also important to note that some fifth graders predicted that machines would act teleologically, at levels similar to that of animals, and that there were condition differences for machines just as there were for plants and animals. This pattern of explanations might seem inconsistent with a biology-based model of teleological action. It is not necessarily a contradiction, however, to simultaneously hold a biologybased model and to predict that a machine would act for something that would benefit it. Machines can embody the goals of their designers, and designers presumably design machines to act to benefit both the designers themselves and their creations. Future research involving a "conflict of interests" between designers and machines might lead to an understanding of why fifth graders predicted that machines would act teleologically.

No matter how fifth graders' conception of machines is ultimately identified, preschoolers' models of teleological action seemed to be quite different from the models employed by fifth graders and adults.
Unlike fifth graders and adults, preschoolers' beliefs in the present study bore a strong similarity to the beliefs of infants and toddlers, who expect that humans (but not nonanimals) will act toward goals (Phillips \& Wellman, 1999; Woodward, 1997), expect that goal-directed objects will act "rationally" (Gergely et al., 1995), and use the goals of humans to infer the humans' mental states (Poulin-Dubois \& Shultz, 1990; Wellman, 1990). Similarly, mental-state explanations for animal behavior (e.g., "The zebras stay together because they like each other") are preferred by 6 - to 8 -year-olds over teleological explanations (e.g., "The zebras stay together because they will be safe from danger"), whereas teleological explanations are preferred over mental-state explanations by 8- to 12-year-olds (Poling \& Evans, in press). The transition from a psychology-based to a biologybased model of teleological action after preschool, then, seems to involve a significant conceptual development toward an abstract understanding of biological principles on the one hand, and a new conceptualization of goals on the other.

Why this transition occurs cannot be concluded based on the available evidence. Several sources of conceptual change come to mind. For one, preschoolers may need only limited direct evidence of nonpsychological teleological action to hold a biologybased model. For example, preschoolers may have never been taught that plants grow toward sunlight, that hearts beat blood to themselves, or that amoebae engulf paramecia. Possibly, such teaching would directly lead to the adoption of a biological understanding of teleological action. On the other hand, such direct input may not appear strange to preschoolers at all, particularly if they take such evidence as proof that plants, hearts, and amoebae have psychological capacities. In any case, the transition from a psychology-based to a biology-based model of teleological action has been established, but requires an explanation.

Although the development of teleological reasoning involves a shift from psychology-based to biology-based models after preschool, this does not necessarily imply that preschoolers lack a theory of biology altogether. Past research has found that preschoolers recognize that plants and animals are uniquely capable of growth, healing, nourishment, and reproduction, without appealing to mere associations or mental capacities (Springer \& Keil, 1989). How do children explain such properties? One possibility is that preschoolers possess an essentialist theory of biology that is neither teleological nor psychological. For example, preschoolers could believe that animals and plants uniquely share "something-they- 
know-not-what" that causes them to grow, heal, nourish themselves, and reproduce. Development of a fully formed theory of biology may involve substituting teleological mechanisms in ever more specific terms for that something-they-know-not-what.

\section{ACKNOWLEDGMENTS}

This study was funded in part by NIMH Grant No. T32MH19102-11 to the first author and NICHD Grant No. HD037142 to the second author. Portions of this study were presented at the Jean Piaget Society Conference, June 1998, Chicago, IL; and the biennial meeting of the Society for Research in Child Development, April 1999, Albuquerque, NM. The authors would like to thank Harold Stevenson, Marilyn Shatz, Robert Siegler, and two anonymous reviewers who provided valuable comments on earlier drafts; Linda Opfer at Forest Road Elementary School; and the faculty and staff at the University of Michigan Children's Center and the Children's Center for Working Families. David Guldmann, Patrick Kiley, and Beth Gewirtzmann also provided helpful assistance with data collection.

\section{ADDRESSES AND AFFILIATIONS}

Corresponding author: John E. Opfer, Department of Psychology, Carnegie Mellon University, Baker Hall 331B, Pittsburgh, PA 15213; e-mail: jopfer@andrew. cmu.edu. Susan A. Gelman is at the University of Michigan, Ann Arbor.

\section{REFERENCES}

Backscheider, A. G., Shatz, M., \& Gelman, S. A. (1993). Preschoolers' ability to distinguish living kinds as a function of regrowth. Child Development, 64, 1242-1257.

Binswanger, H. (1992). Life-based teleology and the foundation of ethics. The Monist, 75, 84-103.

Carey, S. (1985). Conceptual change in childhood. Cambridge, MA: MIT Press.

Carey, S. (1995). On the origins of causal understanding. In S. Sperber, D. Premack, \& A. Premack (Eds.), Causal cognition: A multi-disciplinary debate (pp. 268-308). Cambridge, U.K.: Oxford University Press.

Coley, V. (1995). Emerging differentiation of folkbiology and folkpsychology: Attributions of biological and psychological properties to living things. Child Development, $66,1856-1874$.

Gergely, G., Nadasdy, Z., Csibra, G., \& Biro, S. (1995). Taking the intentional stance at 12 months of age. Cognition, 56, 165-193.

Gutheil, G., Vera, A., \& Keil, F. C. (1998). Do houseflies think? Patterns of induction and biological beliefs in development. Cognition, 66, 33-49.

Hickling, A. K., \& Gelman, S. A. (1995). How does your garden grow? Early conceptualization of seeds and their place in the plant growth cycle. Child Development, 66, 856-876.

Inagaki, K., \& Hatano, G. (1993). Young children's understanding of the mind-body distinction. Child Development, 64, 1534-1549.

Keil, F. C. (1992). The origins of an autonomous biology. In M. A. Gunnar \& M. Maratsos (Eds.), Minnesota symposium on child psychology (Vol. 25). Hillsdale, NJ: Erlbaum.

Keil, F. C. (1994). The birth and nurturance of concepts by domains: The origins and concepts of living things. In L. Hirschfeld \& S. A. Gelman (Eds.), Mapping the mind: Domain-specificity in cognition and culture. New York: Cambridge University Press.

Kelemen, D. (1999). The scope of teleological thinking in preschool children. Cognition, 70, 241-272.

Mayr, E. (1982). The growth of biological thought. Cambridge, MA; Harvard University Press.

Phillips, A., \& Wellman, H. M. (1999, April). Infants' understanding of goal-directed action. Paper presented at the biennial meeting of the Society for Research in Child Development, Albuquerque, NM.

Piaget, J. (1969). The child's conception of physical causality. Totawa, NJ: Littlefield, Adams, \& Co.

Poling, D. A., \& Evans, E. M. (in press). Why do birds of a feather flock together? Developmental change in the use of multiple explanations: Intention, teleology, essentialism. British Journal of Developmental Psychology.

Poulin-Dubois, D., \& Shultz, T. R. (1990). The infant's concept of agency: The distinction between social and nonsocial objects. Journal of Genetic Psychology, 151, 77-90.

Rosenblueth, A., Wiener, N., \& Bigelow, J. (1943). Behavior, purpose, and teleology. Philosophy of Science, 10, 18-24.

Siegler, R. S. (1996). Emerging minds: The process of change in children's thinking. New York: Oxford University Press.

Solomon, G. E. A., \& Cassimatis, N. L. (1999). On facts and conceptual systems: Young children's integration of their understandings of germs and contagion. Developmental Psychology, 35, 113-126.

Spelke, E. S., Phillips, A., \& Woodward, A. L. (1995). Infants' knowledge of object motion and human action. In D. Sperber, D. Premack, \& A. J. Premack (Eds.), Causal cognition: A multidisciplinary debate (pp. 44-78). New York: Oxford University Press.

Springer, K., \& Keil, F. C. (1989). On the development of biologically specific beliefs: The case of inheritance. Child Development, 60, 637-648.

Wellman, H. M. (1990). The child's theory of mind. Cambridge, MA: MIT Press.

Wellman, H. M., \& Gelman, S. A. (1992). Cognitive development: Foundational theories of core domains. Annual Review of Psychology, 43, 337-375.

Woodward, A. (1997, April). What do infants understand about goal-directed action? Paper presented at the Conference on Developmental Processes in Early Social Understanding, Ann Arbor, MI. 